DIVISION OF THE HUMANITIES AND SOCIAL SCIENCES

CALIFORNIA INSTITUTE OF TECHNOLOGY

PASADENA, CALIFORNIA 91125

CONSISTENT REPRESENTATIVE DEMOCRACY

Christopher P. Chambers

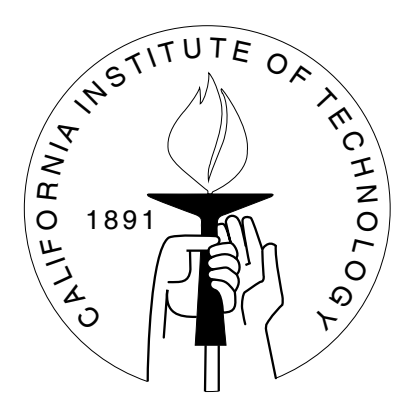

SOCIAL SCIENCE WORKING PAPER 1217 


\title{
Consistent representative democracy
}

\author{
Christopher P. Chambers
}

\begin{abstract}
We study axioms which define "representative democracy" in an environment in which agents vote over a finite set of alternatives. We focus on a property that states that whether votes are aggregated directly or indirectly makes no difference. We call this property representative consistency. Representative consistency formalizes the idea that a voting rule should be immune to gerrymandering. We characterize the class of rules satisfying unanimity, anonymity, and representative consistency. We call these rules "partial priority rules." A partial priority rule can be interpreted as a rule in which each agent can "veto" certain alternatives. We investigate the implications of imposing other axioms to the list specified above. We also study the partial priority rules in the context of specific economic models.
\end{abstract}

JEL classification numbers: D63, D70

Key words: Social choice, representative systems, majority rule, gerrymandering 


\title{
Consistent representative democracy *
}

\author{
Christopher P. Chambers
}

\section{Introduction}

Our objective is to discuss an interpretation of "representative democracy" in a voting model. In so doing, we provide a meaningful axiomatic definition of the meaning of representative.

In binary social choice, the only voting rule which is anonymous, neutral, and positively responsive is majority rule (May's Theorem, [14]). May's theorem provides a normative foundation for majority rule. However, most democracies use some form of representative democracy. Voters are grouped into districts, and each district elects its own representative according to majority rule. These representatives then vote, and majority rule is applied to the votes of the representatives. Such a system is referred to as a "single-member district" system. Typically, single-member district representation results in a different outcome than if majority rule were applied directly to the votes of the voters. The alternative selected depends non-trivially on how voters are grouped into districts, and is thus subject to manipulation by those who design the districts. In common language, we say that "gerrymandering" is possible. Gerrymandering is a common practice in single-member district systems.

A natural question is whether or not there exist voting rules which can never be gerrymandered. Our primary contribution here is twofold. Firstly, we introduce a condition which formalizes the notion that a voting rule should be immune to gerrymandering. To our knowledge, ours is the first work that investigates such an idea and proposes such a notion. Secondly, we investigate the implications of this notion in conjunction with other properties, and characterize all "democratic" voting rules that satisfy it.

Here, we imagine that society's collective goal is to select one element out of a finite set of social alternatives. We suppose that each agent in society submits a vote for one of the alternatives. A social alternative must be selected based only on the observed votes, not taking into account strategic behavior on the part of voters. We study "rules,"

*I would like to thank John Duggan, Larry Epstein, Biung-Ghi Ju, Roger Lagunoff, Leonardo Martinez, Josef Perktold, Francesco Squintani, William Thomson, and Chun-Hsien Yeh for useful conversations. All errors are my own. 
which associate a social alternative with every possible group of voters and list of votes that they may submit. Since a rule can be applied to any group of voters, it can be applied to districts, or it can be applied to society as a whole.

When we say a voting rule is democratic, we mean that it $i$ ) selects any alternative which receives a unanimity of votes and $i i$ ) treats all agents identically. These requirements are captured by the properties unanimity and anonymity, respectively. Unlike May, we make no monotonicity or neutrality assumptions on rules.

We specify notions of "direct democracy" and "indirect democracy." These two notions are discussed with respect to a given rule. In a direct democracy, each member of society votes for an alternative, and an alternative is selected by aggregating these votes according to the rule. In an indirect democracy, agents are partitioned into districts, and each agent votes for an alternative. The rule is then applied within each district, resulting in a "representative vote" for each district. The winning alternative in each district takes all of the votes for that district. Thus, each agent in society is treated as having voted for his district's representative vote. The rule is then applied to the representative votes, leading to a social alternative. We say a rule is a "representative democracy" if direct democracy and indirect democracy lead to the same chosen alternative, independently of the partitioning of agents. The equivalence of direct and indirect vote aggregation is logically independent from the notion of "democracy," so we give it an independent name: representative consistency.

To understand the notion, consider the simple example of a nine-agent society that uses majority rule. The voters in society need to decide whether or not to pass a bill into law. Voters vote for either $Y$ (pass the bill) or $N$ (do not pass the bill). Imagine that the nine tallied votes are $\{Y, Y, N, N, N, N, N, Y, Y\}$-four votes to pass and five votes not to pass. By applying majority rule directly to this vote profile, the bill does not pass into law. But suppose that we consider the following partition of voters: $\{\{Y, Y, N\},\{N, N, N\},\{N, Y, Y\}\}$. Here, the voters have been put into three districts. In each of the first and last district, there are two votes for $Y$ and one for $N$. In either of these districts, $Y$ is selected according to majority rule. Obviously, in the remaining district, where there are three votes for $N, N$ is selected according to majority rule. $Y$ therefore takes the first and last district, and $N$ takes the remaining district. The revised vote profile obtained is $\{Y, Y, Y, N, N, N, Y, Y, Y\}$. But for this revised vote profile, there are six votes for $Y$ and only three for $N$. Thus, under the revised vote profile, the bill passes into law. This example illustrates that majority rule is not representative consistent. ${ }^{1}$

The notion of indirect vote aggregation in social choice is familiar from the works of Murakami [16, 17], Fishburn [11, 12], and Fine [10], although these papers are not concerned with the consistency of indirect vote aggregation and direct vote aggregation. In his famous paper on strategy-proof voting schemes, Moulin [15] also briefly discusses

\footnotetext{
${ }^{1}$ Here, we have not discussed what happens when there is a tie. But clearly, this example shows that majority rule is not representative consistent, no matter how ties are resolved.
} 
indirect voting procedures.

Representative consistency is a strong condition of "gerrymandering-proofness." ${ }^{2}$ It is reasonable to suspect that under a single-member district system, almost any voting rule is susceptible to gerrymandering. Our primary result confirms this intuition. However, the conditions unanimity, anonymity, and representative consistency are compatible, and characterize a class of rules that we believe has not been studied before. These rules are easiest to understand when there are only two alternatives. In this case, one of the alternatives is "special," and the rule chooses the special alternative unless all agents vote for the other alternative. Thus, each agent has a "veto" and can force society to choose the special alternative by voting for it.

These rules can be described in a different way. Think of the two alternatives as being ordered, where the special alternative comes first in the order. Among the alternatives receiving votes, the rule selects the one that comes first in the order. We show that for any finite number of alternatives, each rule satisfying our conditions is identified with a partial order over the set of alternatives. If two alternatives are ranked according to this partial order, then we say that the two alternatives are prioritized. Of these two alternatives, the alternative which comes first according to the partial order has a higher priority. The rule selects the lowest priority alternative which has a priority (weakly) higher than all of the alternatives which receive votes. This alternative need not have been voted for by any of the agents. We call such a rule a "partial priority rule."

Partial priority rules possess the feature that the number of agents who vote for a particular alternative is irrelevant in forming a social decision. This renders them unappealing for many group decision situations. ${ }^{3}$ Thus, in eliciting the general structure of rules that are immune to gerrymandering in single-member districts, we have demonstrated a fundamental impossibility: Unless all agents are given veto power over the same alternatives, a voting rule is susceptible to gerrymandering. Roughly, this impossibility highlights a tradeoff between the amount of "power" possessed by each voter, and the amount of "power" possessed by those in charge of formulating districts.

Partial priority rules are used in real-life environments. A jury must decide between the guilt or the innocence of a suspect. A suspect is innocent unless the jurors unanimously agree on her guilt. The partial order corresponding to this rule ranks "guilty" after "innocent." Suppose jurors can elect to abstain, by voting for an alternative called "hung jury." Here, if all agents agree on guilty, the suspect is guilty. If all agents agree on innocent, the suspect is innocent. If there is not unanimous agreement, or if some

\footnotetext{
${ }^{2}$ Thus, we can think of other notions of a rule being immune to gerrymandering. Specifically, by ignoring strategic effects on the part of voters, we are ruling out the possibility that agents may behave differently when placed in different districts. Such behavior may have the effect of "cancelling out" attempts at gerrymandering. A weaker notion of representative consistency may be desired in this case; such a notion will depend on the assumed equilibrium concept for voter behavior.

${ }^{3}$ They are unappealing for many reasons. For example, Duggan and Martinelli [8] show that unanimity rules (partial priority rules in binary environments) perform poorly in terms of aggregating information.
} 
voter chooses to abstain (by voting "hung jury"), we say that there is a "hung jury." With this rule, both "guilty" and "innocent" are ranked after "hung jury," but "guilty" and "innocent" are incomparable.

Although we view our main result as an impossibility, the class of partial priority rules is nonempty. This allows us to imposes additional properties on rules. One feature of the partial priority rules is that the alternative selected by society need not have received any votes. In the preceding example, if some voters vote guilty, and others vote innocent, then the outcome is "hung jury," even if no agent had voted for a "hung jury." However, for many social decisions, it is unnatural to choose an alternative for which nobody votes. In an election between candidates, a candidate who receives no votes should not be elected. We introduce a condition, called positive vote-share, that requires that an alternative must receive at least one vote to be selected. When coupled with our other axioms, this condition implies that the partial order corresponding to a partial priority rule is a linear order.

We assume no structure on the set of alternatives except that it is finite. In specific examples, however, restrictions on the set of alternatives are warranted. To this end, we study the partial priority rules in a spatial model. Here, the alternatives are exogenously ordered according to some linear order.

We postulate conditions relating to this exogenous order. Betweenness is the requirement that the selected alternative lie between the minimal and maximal votes according to the linear order. If it is implicitly understood that agents have single-peaked preferences over the alternatives and votes are the agents' peaks, betweenness is equivalent to Pareto efficiency. Vote monotonicity states that if the votes of all agents move in a certain direction, then so should the selected alternative. Vote monotonicity can be understood as a simple positive responsiveness condition. Lastly, we study strategy-proofness, assuming that agents have preferences over the alternatives that are single-peaked. Strategyproofness states that when all agents are required to vote for an alternative, it is a dominant strategy for each agent to vote for her peak. When coupled with our other axioms, these axioms lead to three progressively more restrictive classes of rules, which we characterize.

Section 2 discusses the model and the main axioms. Section 3 proves our main theorem and several simple corollaries. Section 4 discusses our theorem in a spatial model. Section 5 concludes.

\section{The model}

\subsection{Preliminaries}

Let $\mathbb{N}$, the set of natural numbers, index a set of "potential agents." Let $\mathcal{N}$ be the set of finite subsets of $\mathbb{N}$. Let $X$ be a finite set. Elements of $X$ are "alternatives," to be 
interpreted as policies, candidates, etc. Each agent submits a "vote," or an element of $X$. A rule is a function $f: \bigcup_{N \in \mathcal{N}} X^{N} \rightarrow X$. Thus, a rule specifies a "representative" alternative for any population of agents and any list of votes these agents may submit.

\subsection{Democracy}

The first property we discuss in this section states that a rule should respect the "will of the people" when this "will" is unambiguous. It is an extremely weak axiom.

For all $N \in \mathcal{N}$ and all $x \in X$, let $x^{N}$ be a vector in $X^{N}$ such that for all $i \in N$, $x_{i}^{N}=x$. For all $N \in \mathcal{N}$, all $y \in X^{N}$, and all $M \subset N$, let $y_{M}$ be the restriction of $y$ to $X^{M}$.

Unanimity: For all $N \in \mathcal{N}$ and all $x \in X, f\left(x^{N}\right)=x$.

The next axiom states that a rule should be ignorant of the names of agents. This is a mild principle of equality, capturing the "one-man, one-vote" principle.

Anonymity: For all $N, N^{\prime} \in \mathcal{N}$ such that $|N|=\left|N^{\prime}\right|$, all bijections $\sigma: N \rightarrow N^{\prime}$, and all $x \in X^{N}$ and $x^{\prime} \in X^{N^{\prime}}$ such that for all $i \in N, x_{i}=x_{\sigma(i)}^{\prime}, f(x)=f\left(x^{\prime}\right)$.

\subsection{Representative consistency}

Informally, representative consistency states that for any population of agents and any collection of votes, it is without loss of generality to partition the set of agents into districts, find the choice for each district, and then treat each district as if each agent in the district had voted for the outcome selected for the district.

Representative consistency: For all $N \in \mathcal{N}$, all partitions $\left\{N_{1}, \ldots, N_{K}\right\}$ of $N$, and all $x \in X^{N}, f(x)=f\left(f\left(x_{N_{1}}\right)^{N_{1}}, \ldots, f\left(x_{N_{K}}\right)^{N_{K}}\right)$.

Following our notation, the vector $\left(f\left(x_{N_{1}}\right)^{N_{1}}, \ldots, f\left(x_{N_{K}}\right)^{N_{K}}\right)$ denotes the vector $y \in$ $X^{N}$, where for all $i \in N_{k}, y_{i}=f\left(x_{N_{k}}\right)$. Under the unanimity principle, representative consistency is equivalent to the stronger statement that for all $N \in \mathcal{N}$, all $M \subset N$, and all $x \in X^{N}, f(x)=f\left(f\left(x_{M}\right)^{M}, x_{N \backslash M}\right)$. This latter version of representative consistency is more useful in the proofs of theorems.

Representative consistency formalizes the idea that the selected alternative should be independent of how the voters are partitioned into districts. While technically a very strong condition, it is a natural formalization of the notion of "gerrymanderingproofness." 
Before proceeding to the main results, we discuss an alternative notion of indirect democracy that may appear to conform more naturally to real-world institutions. In common practice, each district is treated equally, regardless of its size (there is usually some attempt to equalize the populations of districts). This suggests that instead of replacing each agent's vote by the alternative selected for her district, we consider each district as an independent voter, and apply the rule to the vote of the districts. This is essentially the idea employed in the previously discussed works by Murakami [16, 17], Fishburn [11, 12], and Fine [10]. In order to formalize this idea, a rule must be able to take as an argument a vote by a district, where a district is treated as an agent. To accommodate this idea, we suppose that rules $g$ are defined on $X^{\mathbb{N}}$, so that a vote profile only takes into consideration the number of voters voting for each alternative (thus anonymity is presupposed). A notion of representative consistency conforming to the above idea would state that for all $x \in X^{\mathbb{N}}, g(x)=g\left(g\left(x^{1}\right)+g\left(x^{2}\right)+\ldots+g\left(x^{K}\right)\right)$, where $\sum_{j=1}^{K} x^{j}=x$. In this definition, each $j=1, \ldots K$ corresponds to a district. The value $g\left(x^{j}\right)$ is the outcome of the vote in this district. The vector $g\left(x^{1}\right)+g\left(x^{2}\right)+\ldots+$ $g\left(x^{K}\right)$ is thus the collection of representative votes.

This notion is extremely strong. There is little normative justification for treating groups of different sizes equivalently. The notion suggests that a rule is independent not only of how voters are partitioned into districts, but also independent of how much weight each voter is given. Under the implicit hypothesis of anonymity, together with unanimity this notion is easily seen to imply ours.

As mentioned in the introduction, one of our main conclusions is that our axioms actually imply this stronger notion. This is far from obvious; in fact it is not true in a model with an infinite set of alternatives. As a response to the potential criticism that our axiom is not useful because it does not conform to real-world procedures, we offer a weaker version of our notion of representative consistency that better captures the actual procedure. Specifically, in the real-world, while districts are treated equally independently of size, there is usually an attempt to preserve equality of populations across districts.

If districts are required to be equipopulous, then under our notion of representative consistency, there is no loss of generality in treating all districts as if they were single voters (this will become clear in the next section). Under the unanimity and anonymity properties, our notion of representative consistency and the stronger notion discussed above are equivalent when restricting to partitions which equalize the populations of districts. Returning to our model, we suggest an axiom that requires representative consistency to hold only if the partition of agents equalizes the number of agents in each district.

Weak representative consistency: For all $N \in \mathcal{N}$, all partitions $\left\{N_{1}, \ldots, N_{m}\right\}$ of $N$ such that for all $i, j,\left|N_{i}\right|=\left|N_{j}\right|$, and all $x \in X^{N}, f(x)=f\left(f\left(x_{N_{1}}\right)^{N_{1}}, \ldots, f\left(x_{N_{m}}\right)^{N_{m}}\right)$. 
We show in an appendix that under unanimity and anonymity, weak representative consistency implies representative consistency. Thus, this axiom which better fits realworld procedures attains the same results.

\section{$3 \quad$ Results}

\subsection{Preliminaries}

Any anonymous rule can be specified without reference to the specific names of agents. In the proofs of results in which anonymity plays a role, we often exploit this fact without mention, disregarding the variable $N$.

The following axiom can be interpreted as meaning that only the proportions of votes received for each alternative are used in determining the social alternative.

Let $m$ be an integer, let $N \in \mathcal{N}$, and let $x \in X^{N}$. Let $N^{\prime} \in \mathcal{N}$ be such that $\left|N^{\prime}\right|=m|N|$. A vector $x^{\prime} \in X^{N^{\prime}}$ is an m-replica of $\mathbf{x}$ if there exists a partition of $N^{\prime}$ into $m$ sets of size $|N|$, say $\left\{N_{1}, \ldots, N_{m}\right\}$ such that for all $N_{i}$, there exists a bijection $\sigma_{i}: N \rightarrow N_{i}$ so that for all $j \in N, x_{i}=x_{\sigma(i)}^{\prime}$. For all $N \in \mathcal{N}, x \in X^{N}, m \in \mathbb{N}, x^{m}$ denotes an $m$-replica of $x .^{4}$

Replication invariance: Let $m$ be an integer. Let $N \in \mathcal{N}$ and let $x \in X^{N}$. Let $x^{\prime}$ be an $m$-replica of $x$. Then $f\left(x^{\prime}\right)=f(x)$.

The following trivial observation is useful:

Lemma 1: If a rule satisfies unanimity, anonymity, and representative consistency, then it satisfies replication invariance.

Proof: Let $N \in \mathcal{N}$ and let $x \in X^{N}$. Let $x^{\prime}$ be an $m$-replica of $x$. Then by definition of $x^{\prime}, f\left(x^{\prime}\right)=f(\underbrace{x, \ldots, x}_{m})$. By representative consistency, $f\left(x^{\prime}\right)=f(\underbrace{f(x), \ldots, f(x)}_{m|N|})$. By unanimity, $f(\underbrace{f(x), \ldots, f(x)}_{m|N|})=f(x)$. Thus $f\left(x^{\prime}\right)=f(x)$.

Our main result is a characterization of the class of rules satisfying unanimity, anonymity, and representative consistency. The following example illustrates this class in the twoalternative case. The description of the general class follows.

\footnotetext{
${ }^{4}$ There are many $m$-replicas of any given vector.
} 
Example 1: Let $X \equiv\{y, z\}$. Then there are only two rules satisfying unanimity, anonymity, and representative consistency. One such rule selects $f(x)=y$ unless all agents vote for $z$. The other such rule always selects $z$, unless all agents vote for $y$.

We now generalize Example 1 to the case of an arbitrary (finite) number of alternatives. A partial order is a binary relation which is $i$ ) reflexive, $i i$ ) transitive, and $i i i$ ) anti-symmetric. A partial order need not be complete. ${ }^{5}$ For a partial order $\preceq, \prec$ denotes the asymmetric part. ${ }^{6}$ A pair $(Y, \preceq)$ is a partially ordered set if $Y$ is a set and $\preceq$ is a partial order on $Y$. For all $x, y \in Y$, where $(Y, \preceq)$ is a partially ordered set, $x \wedge y \in Y$ is the meet of $\mathbf{x}$ and $\mathbf{y}$ if it is the unique greatest lower bound for $x$ and $y$ according to $\preceq$. Generally, two elements $x, y \in Y$ need not possess a meet. A meet-semilattice is a partially ordered set such that any pair of elements possesses a meet. For more on these definitions, see Birkhoff [4].

Figure 1 displays a typical meet-semilattice. Each meet-semilattice can be pictured as a directed graph with a unique root. Here, $a$ is the root of the graph. An alternative precedes another alternative in terms of the partial order if one can construct a path emanating from the first alternative up the graph to the other alternative. Thus, in Figure 1, $a \preceq c$ as there exists a path going up the graph starting at $a$ and ending at $c$. Moreover, $a \preceq e$. However, the alternatives $b$ and $c$ are unrelated according to $\preceq$, as there exists no directed path between them. The meet of two alternatives is easily found as the highest common predecessor of the two alternatives. Thus, $b \wedge e=a$ in the example, whereas $c \wedge e=c$.

A rule $f$ is a partial priority rule if there exists a partial order $\preceq$ over $X$ so that $(X, \preceq)$ is a meet-semilattice, and for all $N \in \mathcal{N}$ and all $x \in X^{N}, f(x)=\bigwedge_{i \in N} x_{i} \cdot{ }^{7,8}$

Thus, with any partial priority rule, the proportion of votes received for each alternative does not matter. To select an alternative, one only needs to determine which alternatives receive a positive number of votes.

\footnotetext{
${ }^{5}$ Reflexive: For all $x \in X, x \succeq x$.

Transitive: For all $x, y, z \in X$, if $x \succeq y$ and $y \succeq z$. then $x \succeq z$.

Anti-symmetric: For all $x, y \in X$, if $x \succeq y$ and $y \succeq x$, then $x=y$.

Complete: For all $x, y \in X$, either $x \succeq y$ or $y \succeq x$.

${ }^{6}$ That is, $x \succ y$ if and only if $x \succeq y$ is true and $y \succeq x$ is false.

${ }^{7}$ The notation $\bigwedge_{i \in N} x_{i}$ refers to the meet of the set of elements $\left\{x_{i}\right\}_{i \in N}$, which exists for any meetsemilattice.

${ }^{8}$ There is no significance to the fact that partial priority rules select the meet of the alternatives receiving a positive number of votes. Every partial priority rule can equivalently be described through the use of a partially ordered set in which every pair of elements has a join (unique least upper bound), where the rule selects the join of the alternatives receiving a positive number of votes.
} 


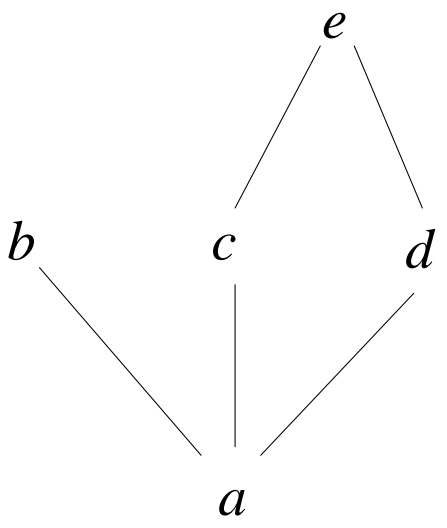

Figure 1: A meet-semilattice

\subsection{Interpreting partial priority rules}

Let $f$ be a partial priority rule with partial order $\preceq$. Let $x, y \in X$ be ordered by $\preceq$, so that $x \preceq y$. We may interpret this statement as reading " $x$ has a higher priority than $y$." In other words, $x$ is "more important" than $y$. This interpretation is possible because $y$ can be selected in place of $x$ only if all agents vote for $y$ in lieu of $x$. Thus, each agent can "veto" $y$ by voting for $x$.

However, not all alternatives need be related by $\preceq$. Suppose in fact that $x$ and $y$ are unrelated according to $\preceq$. Then we may interpret this as meaning that neither $x$ nor $y$ can be deemed a more important alternative. In this case, some "compromise" must be struck between the two. The compromise between $x$ and $y$ is decided using the partial priority. It is the lowest priority alternative which has priority higher than both $x$ and $y$.

\subsection{The main theorem}

The main theorem states that a voting rule is a "representative democracy" if and only if it is a partial priority rule. The proof is constructive and is divided into five steps. Given is a rule satisfying the axioms describing "representative democracy." In the first step, an order on the alternatives is defined from the rule, and it is verified that this order is a partial order. This construction is always valid-even if $X$ is not finite. The most significant obstacle is in showing that this partial order forms a meet-semilattice when coupled with $X$, which need not be true if $X$ is not finite. We establish this fact in Steps 2, 3, and 4. Step 2 shows that the rule is "monotonic" in the partial order. Step 3 shows that for two-agent environments, the selected social alternative lies below the two 
alternatives voted for in terms of the partial order. Step 4 uses Steps 2 and 3 to show that the partial order is actually a meet-semilattice and that for two-agent environments, the rule selects the meet of the alternatives which receive votes. Step 5 establishes that the rule always selects the meet of the alternatives which receive votes.

Theorem 1: A rule satisfies unanimity, anonymity, and representative consistency if and only if it is a partial priority rule.

Proof: If $f$ is a partial priority rule, then it clearly satisfies unanimity, anonymity, and representative consistency. For the other direction, let $f$ be a rule which satisfies unanimity, anonymity, and representative consistency.

\section{Step 1: Construction of the order and verification of its properties.}

For all $x, y \in X$, define $x \preceq y$ if $f(x, y)=x$. Let $x \in X$. By unanimity, $f(x, x)=x$, so that $x \preceq x$. Thus, $\preceq$ is reflexive. Let $x, y \in X$. Suppose $x \preceq y$ and $y \preceq x$. Then by definition, $f(x, y)=x$ and $f(x, y)=y$. Thus, $x=y$ and $\preceq$ is anti-symmetric.

We claim that $\preceq$ is transitive. Let $x, y, z \in X$. Suppose $x \preceq y$ and $y \preceq z$. By replication invariance,

$$
f(x, z)=f(x, x, z, z)
$$

As $x \preceq y$, by definition,

$$
f(x, x, z, z)=f(f(x, y), f(x, y), z, z) .
$$

By representative consistency,

$$
f(f(x, y), f(x, y), z, z)=f(x, y, z, z) .
$$

By representative consistency,

$$
f(x, y, z, z)=f(x, f(y, z), f(y, z), z) .
$$

As $y \preceq z$, by definition,

$$
f(x, f(y, z), f(y, z), z)=f(x, y, y, z) .
$$

By representative consistency,

$$
f(x, y, y, z)=f(x, y, f(y, z), f(y, z)) .
$$

As $y \preceq z$, by definition,

$$
f(x, y, f(y, z), f(y, z))=f(x, y, y, y) .
$$

By representative consistency,

$$
f(x, y, y, y)=f(f(x, y), f(x, y), y, y) .
$$


As $x \preceq y$, by definition,

$$
f(f(x, y), f(x, y), y, y)=f(x, x, y, y) .
$$

By replication invariance,

$$
f(x, x, y, y)=f(x, y)
$$

As $x \preceq y$, by definition,

$$
f(x, y)=x .
$$

Thus, $f(x, z)=x$, so that by definition, $x \preceq z$. Therefore, $\preceq$ is transitive.

\section{Step 2: The rule is monotonic in the partial order.}

We claim that for all $x, y, z \in X$, if $x \preceq y$, then $f(x, z) \preceq f(y, z)$. Thus, let $x, y, z \in X$ and suppose $x \preceq y$. By replication invariance,

$$
f(f(x, z), f(y, z))=f(f(x, z), f(x, z), f(y, z), f(y, z)) .
$$

By representative consistency,

$$
f(f(x, z), f(x, z), f(y, z), f(y, z))=f(x, z, y, z) .
$$

By anonymity, we may rewrite

$$
f(x, z, y, z)=f(x, y, z, z) .
$$

By representative consistency,

$$
f(x, y, z, z)=f(f(x, y), f(x, y), z, z) .
$$

By replication invariance,

$$
f(f(x, y), f(x, y), z, z)=f(f(x, y), z) .
$$

As $x \preceq y$, by definition,

$$
f(f(x, y), z)=f(x, z) .
$$

Therefore,

$$
f(f(x, z), f(y, z))=f(x, z),
$$

so that, by definition, $f(x, z) \preceq f(y, z)$.

Step 3: The rule maps every two element set into an element which precedes them in the partial order.

We claim that for all $x, y \in X, f(x, y) \preceq x$. Let $x, y \in X$. We define an auxiliary function $F$ derived from $f$. Let $P \equiv \mathbb{Q} \cap(0,1)$ (this will be the domain of $F$ ). 
For all $p \in P, p$ can be written as $p \equiv \frac{m(p)}{n(p)}$, where $m(p), n(p) \in \mathbb{N} .{ }^{9}$ For all $p \in P$, define $F(p)=f\left(x^{m(p)}, y^{n(p)-m(p)}\right)$. By replication invariance, which follows from Lemma $1, F$ is well-defined.

We claim that for all $p, p^{\prime} \in P$ and $z \in X$ such that $p<p^{\prime}$, if $F(p)=z$ and $F\left(p^{\prime}\right)=z$, then for all $p^{\prime \prime} \in\left(p, p^{\prime}\right) \cap P, F\left(p^{\prime \prime}\right)=z$.

Thus, let $p, p^{\prime} \in P$ be such that $F(p)=F\left(p^{\prime}\right)=z$. Let $p^{\prime \prime} \in\left(p, p^{\prime}\right) \cap P$. Then there exists $\alpha \in \mathbb{Q}$ such that $p^{\prime \prime}=\alpha p+(1-\alpha) p^{\prime}$. Since $\alpha \in \mathbb{Q}, \alpha=\frac{m(\alpha)}{n(\alpha)}$ for $m(\alpha), n(\alpha) \in \mathbb{N}$. Thus $p^{\prime \prime}=\frac{m(\alpha)}{n(\alpha)} \frac{m(p)}{n(p)}+\frac{(n(\alpha)-m(\alpha))}{n(\alpha)} \frac{m\left(p^{\prime}\right)}{n\left(p^{\prime}\right)}$. We can write $p^{\prime \prime}=\frac{m(\alpha) m(p) n\left(p^{\prime}\right)+(n(\alpha)-m(\alpha)) m\left(p^{\prime}\right) n(p)}{n(\alpha) n(p) n\left(p^{\prime}\right)}$. Thus, by appropriately arranging terms, $F\left(p^{\prime \prime}\right)$ is equal to:

$$
f\left(\left(x^{m(p)}, y^{n(p)-m(p)}\right)^{n\left(p^{\prime}\right) m(\alpha)},\left(x^{m\left(p^{\prime}\right)}, y^{n\left(p^{\prime}\right)-m\left(p^{\prime}\right)}\right)^{n(p)(n(\alpha)-m(\alpha))}\right)
$$

By assumption, $f\left(x^{m(p)}, y^{n(p)-m(p)}\right)=f\left(x^{m\left(p^{\prime}\right)}, y^{n\left(p^{\prime}\right)-m\left(p^{\prime}\right)}\right)=z$, so that by representative consistency, (1) is equal to $f\left(z^{n(p) n\left(p^{\prime}\right) m(\alpha)}, z^{n\left(p^{\prime}\right) n(p)(n(\alpha)-m(\alpha))}\right)$. By unanimity, $f\left(z^{n(p) n\left(p^{\prime}\right) m(\alpha)}, z^{n\left(p^{\prime}\right) n(p)(n(\alpha)-m(\alpha))}\right)=z$. Therefore, $F\left(p^{\prime \prime}\right)=z$. Hence, $F$ is "convex."

We next show that $F$ is constant on $P$. Since $X$ is finite, there exists $z \in X$ and a sequence $\left\{p^{n}\right\}_{n=1}^{\infty}$ of elements of $P$ such that $p^{n} \rightarrow 1$, for which $F\left(p^{n}\right)=z$ for all $n$. Conclude by the previous paragraph (using the "convexity" of $F$ ), that there exists $p^{*}<1$ such that for all $p \in\left(p^{*}, 1\right) \cap P, F(p)=z$.

We claim that for all $p, q \in P$ and all $\alpha \in(0,1) \cap \mathbb{Q}$, if $F(p)=F(q)$, then $F(\alpha p)=$ $F(\alpha q)$. Thus, let $p, q, \alpha$ satisfy these hypotheses and suppose $F(p)=F(q)=z$. Write $p=\frac{m(p)}{n(p)}, q=\frac{m(q)}{n(q)}$, and $\alpha=\frac{m(\alpha)}{n(\alpha)}$, where $m(p), n(p), m(q), n(q), m(\alpha)$, and $n(\alpha) \in$ $\mathbb{N}$. By definition, $F(p)=f\left(x^{m(p)}, y^{n(p)-m(p)}\right)$ and $F(q)=f\left(x^{m(q)}, y^{n(q)-m(q)}\right)$. By appropriately arranging terms,

$$
F(\alpha p)=f\left(\left(x^{m(p)}, y^{n(p)-m(p)}\right)^{m(\alpha)}, y^{n(p)(n(\alpha)-m(\alpha))}\right)
$$

and

$$
F(\alpha q)=f\left(\left(x^{m(q)}, y^{n(q)-m(q)}\right)^{m(\alpha)}, y^{n(q)(n(\alpha)-m(\alpha))}\right) .
$$

By replication invariance, we obtain (by replicating $n(q)$ times the inside of (2))

$$
F(\alpha p)=f\left(\left(x^{m(p)}, y^{n(p)-m(p)}\right)^{n(q) m(\alpha)}, y^{n(p) n(q)(n(\alpha)-m(\alpha))}\right)
$$

and (by replicating $n(p)$ the inside of $(3)$ )

$$
F(\alpha q)=f\left(\left(x^{m(q)}, y^{n(q)-m(q)}\right)^{n(p) m(\alpha)}, y^{n(p) n(q)(n(\alpha)-m(\alpha))}\right) .
$$

\footnotetext{
${ }^{9}$ This representation of $p$ is obviously not unique.
} 
By representative consistency,

$$
F(\alpha p)=f\left(f\left(x^{m(p)}, y^{n(p)-n(p)}\right)^{n(p) n(q) m(\alpha)}, y^{n(p) n(q)(n(\alpha)-m(\alpha))}\right)
$$

and

$$
F(\alpha q)=f\left(f\left(x^{m(q)}, y^{n(q)-m(q)}\right)^{n(p) n(q) m(\alpha)}, y^{n(p) n(q)(n(\alpha)-m(\alpha))}\right) .
$$

As $f\left(x^{m(p)}, y^{n(p)-m(p)}\right)=f\left(x^{m(q)}, y^{n(q)-m(q)}\right)=z$,

$$
F(\alpha p)=f\left(z^{n(p) n(q) m(\alpha)}, y^{n(p) n(q)(n(\alpha)-m(\alpha))}\right)
$$

and

$$
F(\alpha q)=f\left(z^{n(p) n(q) m(\alpha)}, y^{n(p) n(q)(n(\alpha)-m(\alpha))}\right) .
$$

Thus $F(\alpha p)=F(\alpha q)$.

Let $q^{*} \in P$ satisfy $q^{*} \in\left(p^{*}, 1\right)$. Then $F\left(q^{*}\right)=z$ and $F\left(p^{*}\right)=z$. Moreover, there exists $\alpha^{*} \in(0,1) \cap \mathbb{Q}$ such that $p^{*}=\alpha^{*} q^{*}$. For all $k=1, \ldots, \infty$, let $q_{k} \equiv\left(\alpha^{*}\right)^{k} q^{*}$. For all $k, q_{k}>0$. As $\alpha^{*}<1, q_{k} \rightarrow 0$.

We claim that for all $k=1, \ldots, \infty, F\left(q_{k}\right)=z$. By definition, $F\left(q_{0}\right)=F\left(q_{1}\right)=z$. Proceed by induction. Let $K \in \mathbb{N}$ such that $K \geq 2$. Suppose that for all $k<K$, $F\left(q_{k}\right)=z$. We show that $F\left(q_{K}\right)=z$. By definition, $F\left(q_{K}\right)=F\left(\alpha^{*} q_{K-1}\right)$. By the induction hypothesis, $F\left(q_{K-1}\right)=F\left(q_{K-2}\right)=z$, so that by the result in the previous paragraph, $F\left(\alpha^{*} q_{K-1}\right)=F\left(\alpha^{*} q_{K-2}\right)$. By definition, $F\left(\alpha^{*} q_{K-2}\right)=F\left(q_{K-1}\right)=z$, so that $F\left(q_{K}\right)=F\left(\alpha^{*} q_{K-1}\right)=z$. Thus, for all $k, F\left(q_{k}\right)=z$. By the "convexity" of $F$, we conclude that $F$ is constant and equal to $z$ on $(0,1)$.

Lastly, we establish that $f(x, y) \preceq x$. By the symmetry of this statement, we also conclude that $f(x, y) \preceq y$. By replication invariance,

$$
f(f(x, y), x)=f(f(x, y), f(x, y), x, x) .
$$

By representative consistency,

$$
f(f(x, y), f(x, y), x, x)=f(y, x, x, x) .
$$

By definition,

$$
f(y, x, x, x)=F(3 / 4) .
$$

By the fact that $F$ is constant on $P$,

$$
F(3 / 4)=F(1 / 2) .
$$

By definition,

$$
F(1 / 2)=f(x, y) .
$$

Thus, $f(f(x, y), x)=f(x, y)$, so that by definition, $f(x, y) \preceq x$. 


\section{Step 4: For two agents, the rule chooses the meet of the alternatives receiving votes}

Let $x, y \in X$. We claim that $f(x, y)$ is the unique greatest lower bound for $x$ and $y$ according to $\preceq$. By Step 3, $f(x, y)$ is a lower bound for $x$ and $y$. Suppose there exists another lower bound for $x$ and $y$, say, $z$. Then, by definition, $z \preceq x$ and $z \preceq y$. By Step 2 , as $z \preceq y, f(x, z) \preceq f(x, y)$. As $z \preceq x$, by definition, $f(x, z)=z$, so that $z \preceq f(x, y)$. Thus, by the anti-symmetry of $\preceq, f(x, y)$ is the unique greatest lower bound for $x$ and $y$. Therefore, $f(x, y)=x \wedge y$. Thus, $(X, \preceq)$ is a meet-semilattice. Therefore, for all $N \in \mathcal{N}$ and all $x \in X^{N}, \bigwedge_{i \in N} x_{i}$ is well-defined (as $\wedge$ is associative-see Birkhoff [4], p. 8-10).

\section{Step 5: Extending the result to arbitrary finite numbers of agents}

We establish that for all $N \in \mathcal{N}$ and all $x \in X^{N}, f(x)=\bigwedge_{i \in N} x_{i}$. The proof proceeds by induction on the cardinality of the set of agents $N$. Suppose that $|N|=1$. By unanimity, $f(x)=x=\bigwedge_{i \in N} x_{i}$. Suppose that $|N|=2$. Then by Step $4, f(x)=\bigwedge_{i \in N} x_{i}$.

Let $K \in \mathbb{N}$ such that $K>2$. Suppose that for all $N \in \mathcal{N}$ such that $|N|<K$ and all $x \in X^{N}, f(x)=\bigwedge_{i \in N} x_{i}$. Let $N^{*} \in \mathcal{N}$ such that $\left|N^{*}\right|=K$. Without loss of generality, write $N^{*} \equiv\{1, \ldots, K\}$. Let $x \in X^{N^{*}}$. By representative consistency,

$$
f\left(x_{1}, \ldots, x_{K}\right)=f\left(f\left(x_{1}, \ldots, x_{K-1}\right), \ldots, f\left(x_{1}, \ldots, x_{K-1}\right), x_{K}\right) .
$$

By the induction hypothesis,

$$
f\left(f\left(x_{1}, \ldots, x_{K-1}\right), \ldots, f\left(x_{1}, \ldots, x_{K-1}\right), x_{K}\right)=f\left(\bigwedge_{i=1}^{K-1} x_{i}, \ldots, \bigwedge_{i=1}^{K-1} x_{i}, x_{K}\right) .
$$

By representative consistency (using the fact that $K>3$ ),

$$
f\left(\bigwedge_{i=1}^{K-1} x_{i}, \ldots, \bigwedge_{i=1}^{K-1} x_{i}, x_{K}\right)=f\left(\begin{array}{c}
\bigwedge_{i=1}^{K-1} x_{i}, f\left(\bigwedge_{i=1}^{K-1} x_{i}, \ldots, \bigwedge_{i=1}^{K-1} x_{i}, x_{K}\right), \ldots \\
f\left(\bigwedge_{i=1}^{K-1} x_{i}, \ldots, \bigwedge_{i=1}^{K-1} x_{i}, x_{K}\right)
\end{array}\right)
$$

By the induction hypothesis, the previous expression is equal to

$$
f\left(\bigwedge_{i=1}^{K-1} x_{i}, \bigwedge_{i=1}^{K} x_{i}, \ldots, \bigwedge_{i=1}^{K} x_{i}\right)
$$

By representative consistency,

$$
f\left(\bigwedge_{i=1}^{K-1} x_{i}, \bigwedge_{i=1}^{K} x_{i}, \ldots, \bigwedge_{i=1}^{K} x_{i}\right)=f\left(\begin{array}{c}
f\left(\bigwedge_{i=1}^{K-1} x_{i}, \bigwedge_{i=1}^{K} x_{i}\right), f\left(\bigwedge_{i=1}^{K-1} x_{i}, \bigwedge_{i=1}^{K} x_{i}\right) \\
\bigwedge_{i=1}^{K} x_{i}, \ldots, \bigwedge_{i=1}^{K} x_{i}
\end{array}\right)
$$

By the induction hypothesis, the previous expression is equal to

$$
f\left(\bigwedge_{i=1}^{K} x_{i}, \ldots, \bigwedge_{i=1}^{K} x_{i}\right)
$$


By unanimity, $f\left(\bigwedge_{i=1}^{K} x_{i}, \ldots, \bigwedge_{i=1}^{K} x_{i}\right)=\bigwedge_{i=1}^{K} x_{i}$. Therefore, our induction hypothesis is true.

The following is a natural example of a partial priority rule.

Example 2: Sets of alternatives Let $A$ be a finite set, perhaps representing a set of public projects. Voters need to determine which public projects to undertake. To this end, we study the power set of $A$. Thus, $2^{A}$ is naturally ordered by set inclusion, and it is clear that $\left(2^{A}, C\right)$ forms a meet-semilattice, where for all $B, C \in 2^{A}$, $B \wedge C=B \cap C$. Define $f$ so that, for all $N \in \mathcal{N}$ and all $B \in\left(2^{A}\right)^{N}, f\left(\left(B_{i}\right)_{i \in N}\right) \equiv$ $\bigcap_{i \in N} B_{i}$. Thus, $f$ selects all "alternatives" which are voted for by all agents.

The partial priority rules satisfy the three axioms no matter what the cardinality of $X$ is. But in order for them to be the only rules satisfying the three axioms, it is necessary that $X$ be finite. Here is an example of a set $X$ which is countably infinite, and a rule $f$ on $X$ satisfying the three axioms which is not a partial priority rule.

Example 3: A countably infinite set of alternatives Let $X$ be countably infinite. Let $r: \mathbb{Q} \rightarrow X$ be a bijection (such a bijection exists as $X$ and $\mathbb{Q}$ have the same cardinality). Define $f: \bigcup_{N \in \mathcal{N}} X^{N} \rightarrow X$ as

$$
f\left(\left(x_{i}\right)_{i \in N}\right) \equiv r\left(\frac{\sum_{i \in N} r^{-1}\left(x_{i}\right)}{|N|}\right) .
$$

It is simple to verify that $f$ satisfies the three axioms listed in the theorem. To see that $f$ is not a partial priority rule, let $N$ be a two-agent set and $N^{\prime}$ be a three agent set. Let $x, y \in X$ such that $x \neq y$. Then $(x, y) \in X^{N}$ and $(x, x, y) \in X^{N^{\prime}}$. Suppose $f$ is a partial priority rule. Then $f(x, y)=x \wedge y=x \wedge x \wedge y=f(x, x, y)$. By definition, $f(x, y)=r\left(\frac{r^{-1}(x)+r^{-1}(y)}{2}\right)$ and $f(x, x, y)=r\left(\frac{2 r^{-1}(x)+r^{-1}(y)}{3}\right)$. As $r$ is a bijection, conclude $\frac{r^{-1}(x)+r^{-1}(y)}{2}=\frac{2 r^{-1}(x)+r^{-1}(y)}{3}$. Simple algebra allows us to conclude $r^{-1}(x)=r^{-1}(y)$. As $r$ is a bijection, $x=y$. But we supposed $x \neq y$, a contradiction. A partial order $\preceq$ can be defined from $f$ as in the proof of Theorem 1, Step 1. However, it is immediate that if $x \neq y$, then $f(x, y) \notin\{x, y\}$. This means that no pairs of alternatives are comparable, so that $\preceq$ is the trivial partial order in which all alternatives are only comparable to themselves. Thus, $(X, \preceq)$ does not form a meet-semilattice. ${ }^{10,11}$

\footnotetext{
${ }^{10}$ We are able to describe a large class of rules on infinite sets of alternatives which satisfy our three axioms. Essentially, these rules work by composing the partial priority rules with rules similar to those described in the example.

${ }^{11}$ Infinite sets of alternatives are useful in modelling "proportional representation." Instead of voting for alternatives, agents vote for lotteries over the alternatives. The interpretation of a lottery is that the governing body is composed of proportions of agents who support each particular alternative.
} 


\subsection{Positive vote-share and priority orderings}

A partial priority rule cannot generally be interpreted as prioritizing all alternatives. Here, we introduce a condition which implies that the partial order underlying a partial priority rule is a linear order. It states that an alternative cannot be selected without receiving any votes. It is natural to impose when $X$ is a set of candidates, for example.

Positive vote-share: For all $N \in \mathcal{N}$ and all $x \in X^{N}, f(x) \in\left\{x_{i}\right\}_{i \in N}$.

The following corollary states that the partial priority rules satisfying positive voteshare are exactly those partial priority rules which prioritize all alternatives. ${ }^{12}$

Corollary 1: A rule $f$ satisfies unanimity, anonymity, representative consistency, and positive vote-share if and only if there exists a linear order $\preceq$ over $X$ such that for all $N \in \mathcal{N}$ and all $x \in X^{N}, f(x) \equiv \min _{\preceq}\left\{x_{i}\right\}_{i \in N}$.

Proof: It is simple to verify that the axioms are satisfied by any such rule.

For the other direction, let $\preceq$ be the partial order constructed from $f$ in Theorem 1 . For all $x, y \in X$, positive vote-share implies that $f(x, y) \in\{x, y\}$. By definition of $\preceq$, either $x \preceq y$ or $y \preceq x$. Hence $\preceq$ is complete, so that it is a linear order.

The preceding corollary can be proved directly without referring to Theorem 1 . In fact, a crucial step in the proof of Theorem 1 relies on the fact that $X$ is finite. However, the preceding corollary can be proved even without the finiteness of $X$. The reasoning is simple. In the proof of the main theorem, we define the partial order over $X$ by $x \preceq y$ if $f(x, y)=x$. However, we had to determine that $(X, \preceq)$ is a meet-semilattice (Step 2, Step 3 , and Step 4$)$. When positive vote-share is satisfied, $(X, \preceq)$ is easily seen to be a meet-semilattice, as $\preceq$ is complete.

\section{The spatial model}

As noted, the partial order corresponding to a partial priority rule is interpreted as a priority of alternatives and not as a way of relating the similarity of alternatives. However, there is often a natural, exogenous way of ordering the set of alternatives. In the remainder of this section, we suppose $X$ is exogenously endowed with a linear order $\leq^{*}$. We interpret $\leq^{*}$ as ordering the alternatives by some attribute in a fashion agreed upon by all potential agents. Thus, for all $x, y, z \in X, x \leq^{*} y \leq^{*} z$ is to be read as " $y$ is more similar to $x$ than $z$ is" in terms of the attribute.

The analysis of this section leads to three progressively more restrictive classes of rules.

\footnotetext{
${ }^{12} \mathrm{~A}$ linear order is any binary relation which is $i$ ) complete, $\left.i i\right)$ transitive, and iii) anti-symmetric.
} 


\subsection{Formal notions of compromise}

The first condition states that the selected social alternative should be a "compromise" amongst the agents' votes, in the weak sense that it lies between the minimal and maximal votes according to $\leq^{*}$. As mentioned in the Introduction, if agents possess single-peaked preferences over $X$ (to be defined formally below), betweenness is equivalent to requiring that a rule select Pareto efficient alternatives.

Betweenness: For all $N \in \mathcal{N}$ and all $x \in X^{N}, \min _{\leq^{*}}\left\{x_{i}\right\}_{i \in N} \leq^{*} f(x) \leq^{*} \max _{\leq^{*}}\left\{x_{i}\right\}_{i \in N^{*}}$.

The next condition is a weak monotonicity condition. If all agents' votes move in a certain direction, so should the selected social alternative. This is a simple condition reflecting the fact that the selected social alternative is "representative" of the agents' votes.

Vote monotonicity: For all $N \in \mathcal{N}$ and all $x, y \in X^{N}$, if for all $i \in N, x_{i} \leq^{*} y_{i}$, then $f(x) \leq^{*} f(y)$.

The following lemmas will be useful.

Lemma 2: If a rule satisfies betweenness, then it satisfies unanimity.

Proof: Let $f$ be a rule that satisfies betweenness. Let $N \in \mathcal{N}$ and let $x \in X$. Then $\min _{\leq^{*}}\left\{x_{i}^{N}\right\}_{i \in N}=x$ and $\max _{\leq^{*}}\left\{x_{i}^{N}\right\}_{i \in N}=x$. By betweenness, $x \leq^{*} f\left(x^{N}\right) \leq^{*} x$. As $\leq^{*}$ is anti-symmetric, $f\left(x^{N}\right)=x$. Therefore, $f$ satisfies unanimity.

Lemma 3: If a rule satisfies unanimity and vote monotonicity, then it satisfies betweenness.

Proof: Let $f$ be a rule that satisfies unanimity and vote monotonicity. Let $N \in$ $\mathcal{N}$ and let $x \in X^{N}$. Then for all $i \in N, \min _{\leq^{*}}\left\{x_{i}\right\}_{i \in N} \leq^{*} x_{i} \leq^{*} \max _{\leq^{*}}\left\{x_{i}\right\}_{i \in N^{*}}$. By unanimity, $f\left(\left(\min _{\leq^{*}}\left\{x_{i}\right\}_{i \in N}\right)^{N}\right)=\min _{\leq^{*}}\left\{x_{i}\right\}_{i \in N}$ and $f\left(\left(\max _{\leq^{*}}\left\{x_{i}\right\}_{i \in N}\right)^{N}\right)=$ $\max _{\leq^{*}}\left\{x_{i}\right\}_{i \in N^{*}}$. Thus, by vote monotonicity, $\min _{\leq^{*}}\left\{x_{i}\right\}_{i \in N} \leq^{*} f(x)$ and $f(x) \leq^{*}$ $\max _{\leq *}\left\{x_{i}\right\}_{i \in N}$. Thus $f$ satisfies betweenness.

\subsection{Results on compromise}

We begin this section by defining two subclasses of the partial priority rules, which depend on the linear order $\leq *$.

Say that a rule $f$ is an interval partial priority rule if it is a partial priority rule and for all $x, y, z \in X$, if $y, z \geq^{*} x$, then $y \wedge z \geq^{*} x$, and if $y, z \leq^{*} x$, then $y \wedge z \leq^{*} x$. 


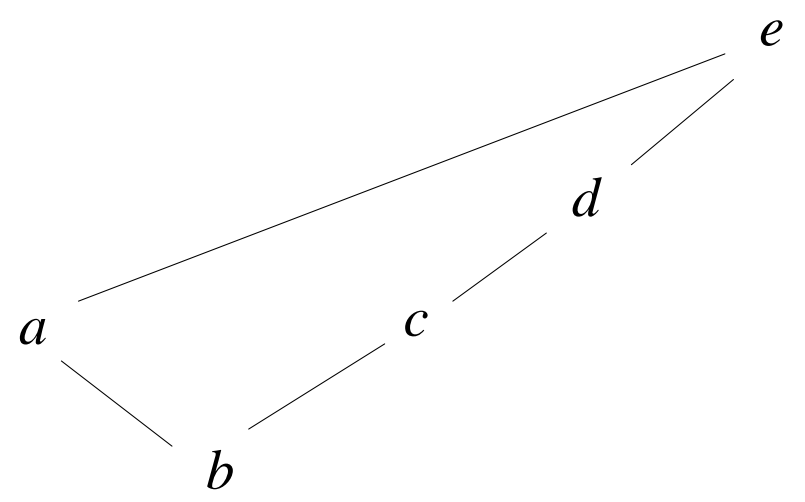

Figure 2: An interval partial priority rule

Thus, a partial priority rule is an interval partial priority rule if the weak lower and upper contour sets of any alternative according to $\leq^{*}$ are themselves meet-semilattices under the partial order induced by $\preceq$. The term "interval" refers to the fact that restricted to any interval according to $\leq^{*}$, the rule is still a partial priority rule. Say that a rule $f$ is a separating partial priority rule if it is an interval partial priority rule such that for all $x, y, z \in X$, if $x \leq^{*} y \leq^{*} z$ and $x, z \succeq y$, then $x \wedge z=y$. Thus, an interval partial priority rule is a separating partial priority rule if the weak lower and upper contour sets of any alternative according to $\leq *$ never "intersect" at any element greater than the alternative itself. The term "separating" refers to the fact that these contour sets never intersect.

Figure 2 displays the meet-semilattice corresponding to a typical interval partial priority rule under the presumption that $a \leq^{*} b \leq^{*} c \leq^{*} d \leq^{*} e$. The general structure of such rules can be grasped from this diagram. The main characteristic of such a rule is that there are at most two branches emanating from any alternative. If there are two branches, one must branch into the upper contour set of the alternative, and the other must branch into the lower contour set (as in the diagram, the two branches emanating from $b$ branch into the two different contour sets). These two branches are allowed to rejoin. It is clear that the meet-semilattice in Figure 2 does not correspond to a separating partial priority rule, as $a \leq^{*} b \leq^{*} e$ and $a, e \succeq b$, yet $a \wedge e=a$.

Figure 3 displays the meet-semilattice corresponding to a typical separating partial priority rule. The general structure of separating partial priority rules can be understood from this diagram. Note that the alternatives $a, b, c, d$, and $e$ are ordered from "left" to "right." Moreover, at most two branches emanate from any given alternative. The last important characteristic is that for any alternative, one can draw an imaginary vertical 


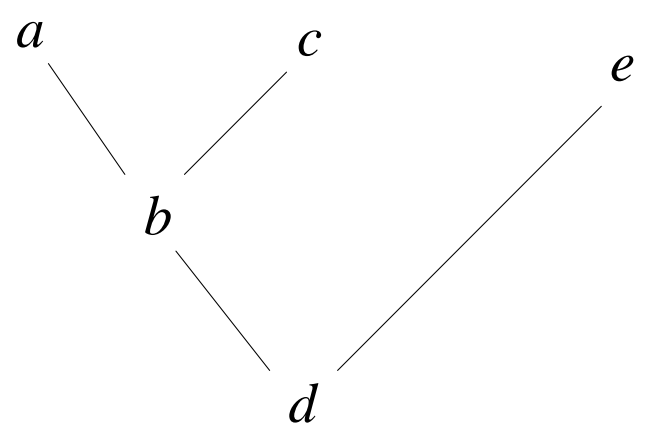

Figure 3: A separating partial priority rule

line branching up from the alternative which never crosses the graph. Thus, the graph corresponding to a separating partial priority rule is a system of interlocking " $\mathrm{y}$ "-shapes. Such a graph looks like an upside-down game tree in which at any given point, there are only two possible moves.

Theorem 2: A rule satisfies betweenness, anonymity, and representative consistency if and only if it is an interval partial priority rule.

Proof: Suppose $f$ is an interval partial priority rule. We already know that it satisfies anonymity and representative consistency, so we will show that it satisfies betweenness. Thus, let $N \in \mathcal{N}$ and $x \in X^{N}$. As $f$ is an interval partial priority rule, and since for all $i \in N, \min _{\leq^{*}}\left\{x_{i}\right\}_{i \in N} \leq^{*} x_{i}$, we obtain $\min _{\leq *}\left\{x_{i}\right\}_{i \in N} \leq^{*} \bigwedge_{i \in N} x_{i}$. A similar expression holds for $\max _{\leq *}\left\{x_{i}\right\}_{i \in N}$, so that $f$ satisfies betweenness.

Now suppose that $f$ is a rule satisfying the axioms. By Lemma 2, we know that it is a partial priority rule. We will verify that $f$ is an interval partial priority rule. Let $x, y, z \in X$, and suppose that $y, z \geq^{*} x$. Without loss of generality, assume that $y \geq^{*} z \geq^{*} x$. By betweenness, $f(y, z) \geq^{*} z$. By the transitivity of $\leq^{*}, f(y, z) \geq^{*} x$. By definition, $f(y, z)=y \wedge z$. Thus, $y \wedge z z^{*} x$. A similar statement holds for the lower contour set of $x$.

Theorem 3 demonstrates that a partial priority rule is vote monotonic if and only if it is a separating partial priority rule.

Theorem 3: A rule satisfies unanimity, anonymity, representative consistency, and vote monotonicity if and only if it is a separating partial priority rule. 
Proof: By Lemma 3 and Theorem 2, if $f$ satisfies the axioms, it is an interval partial priority rule. Let $x, y, z \in X$ satisfy $x \leq^{*} y \leq^{*} z$ and $x, z \succeq y$. By vote monotonicity, $x \wedge z=f(x, z) \leq^{*} f(y, z)=y$ and $x \wedge z=f(x, z) \geq^{*} f(x, y)=y$. By the anti-symmetry of $\leq^{*}, x \wedge z=y$. Thus, $f$ is a separating partial priority rule.

For the other direction, let $f$ be a separating partial priority rule. We will show that it is vote monotonic.

Let $\preceq$ be the partial order associated with $f$. Say a partial priority rule $f$ satisfies condition $(*)$ if for all $x, y, z \in X$, if $x \leq^{*} y$, then $f(x, z) \leq^{*} f(y, z)$. We claim that if a partial priority rule satisfies condition $(*)$, then it is vote monotonic. To see why, suppose $(*)$ is true. Let $N \in \mathcal{N}$, and let $x, y \in X^{N}$. Suppose that for all $i \in N$, $x_{i} \leq^{*} y_{i}$. Suppose, without loss of generality, that $N$ can be ordered as $N \equiv\{1, \ldots, n\}$. For all $m=1, \ldots, n$, let $y_{m} x \equiv\left(y_{1}, \ldots, y_{m}, x_{m+1}, \ldots, x_{n}\right)$. We show by induction that for all $m=1, \ldots, n, f(x) \leq^{*} f\left(y_{m} x\right)$. Let $m=1$. Then $x_{1} \leq^{*} y_{1}$. By condition $(*)$, $x_{1} \wedge\left(\bigwedge_{i=2}^{n} x_{i}\right) \leq^{*} y_{1} \wedge\left(\bigwedge_{i=2}^{n} x_{i}\right)$. Thus, $f(x) \leq^{*} f\left(y_{1} x\right)$. Let $M \leq n$ be some integer. Now, suppose that for all $m<M, f(x) \leq^{*} f\left(y_{m} x\right)$. By assumption, $x_{M} \leq^{*} y_{M}$. By condition $(*)$,

$$
\begin{aligned}
x_{M} & \wedge\left(\left(\bigwedge_{i=1}^{M-1} y_{i}\right) \wedge\left(\bigwedge_{i=M+1}^{n} x_{i}\right)\right) \\
\leq{ }^{*} y_{M} & \wedge\left(\left(\bigwedge_{i=1}^{M-1} y_{i}\right) \wedge\left(\bigwedge_{i=M+1}^{n} x_{i}\right)\right) .
\end{aligned}
$$

The left-hand side of this expression is $f\left(y_{M-1} x\right)$, and the right-hand side is $f\left(y_{M} x\right)$. Thus, $f\left(y_{M-1} x\right) \leq^{*} f\left(y_{M} x\right)$. By transitivity of $\leq^{*}, f(x) \leq^{*} f\left(y_{M-1} x\right) \leq^{*} f\left(y_{M} x\right)$ implies $f(x) \leq^{*} f\left(y_{M} x\right)$. Thus, for all $m=1, \ldots, n, f(x) \leq^{*} f\left(y_{m} x\right)$. In particular, if $m=n$, then $y_{n} x=y$ and $f(x) \leq^{*} f(y)$.

We now show that if a rule is a separating partial priority rule, then it satisfies condition $(*)$. Let $x, y, z \in X$, and suppose that $x \leq^{*} y$. There are three possible cases.

a) $y \leq^{*} z$ : In this case, as $f$ is a separating partial priority rule, it is an interval partial priority rule. Thus, $y \leq^{*} y \wedge z \leq^{*} z$. If $x \wedge z \leq^{*} y \wedge z$, we are done. Otherwise, $y \wedge z \leq^{*} x \wedge z$. As $f$ is an interval partial priority rule, $y \wedge z \leq^{*}(y \wedge z) \wedge(x \wedge z) \leq^{*} x \wedge z$. As $(y \wedge z) \wedge(x \wedge z)=x \wedge y \wedge z, y \leq^{*} x \wedge y \wedge z \leq^{*} z$. By definition, $y, z \succeq x \wedge y \wedge z$. As $f$ is a separating partial priority rule, conclude $y \wedge z=x \wedge(y \wedge z)$. Thus, by definition, $y \wedge z \preceq x$. Thus, $x, z \succeq y \wedge z$ and $x \leq^{*} y \wedge z \leq^{*} z$. As $f$ is a separating partial priority rule, conclude $x \wedge z=y \wedge z$. Thus, $x \wedge z \leq^{*} y \wedge z$.

b) $x \leq^{*} z \leq^{*} y$ : In this case, as $f$ is an interval partial priority rule, $x \leq^{*} x \wedge z \leq^{*}$ $z \leq^{*} y \wedge z \leq^{*} y$, so that $x \wedge z \leq^{*} y \wedge z$.

c) $z \leq^{*} x$ : This case is symmetric to case a). 


\subsection{Strategic considerations in the spatial model}

We now study a domain of preferences over $X$. Say a binary relation $R$ on $X$ is singlepeaked if there exists $x(R) \in X$ such that for all $x, y \in X$, if $x(R) \leq^{*} x<^{*} y$ or if $x(R) \geq^{*} x>^{*} y, x P y \cdot{ }^{13}$ Let $S P\left(X, \leq^{*}\right)$ be the set of all single-peaked binary relations for the linearly ordered set $\left(X, \leq^{*}\right) .^{14}$

We ask which partial priority rules satisfy the following condition, which states that if votes are taken to be agents' peaks, then no agent can ever benefit by lying about his peak. This condition was first studied in this model by Moulin [15].

Strategy-proofness: For all $N \in \mathcal{N}$, all $R \in S P\left(X, \leq^{*}\right)^{N}$, all $j \in N$, and all $R_{j}^{\prime} \in$ $S P\left(X, \leq^{*}\right), f\left(\left(x\left(R_{i}\right)\right)_{i \in N}\right) R_{j} f\left(\left(x\left(R_{i}\right)\right)_{i \in N \backslash\{j\}}, x\left(R_{j}^{\prime}\right)\right)$.

Say a partial priority rule $f$ is a target rule if there exists $x^{*} \in X$ such that for all $y, z \in X, i) z \leq^{*} y \leq^{*} x^{*}$ implies $z \succeq y$, ii) $x^{*} \leq^{*} y \leq^{*} z$ implies $z \succeq y$, and iii) $y \leq^{*} x^{*} \leq^{*} z$ implies $y \wedge z=x^{*}$. Thus, a target rule is a partial priority rule with two branches, rooted at $x^{*}$, one of which agrees with the order $\leq^{*}$, the other of which is the opposite of the order $\leq^{*}$. Target rules were first discussed by Thomson and Thomson and Ching [20,21] using a different set of axioms. The terminology belongs to them. The word "target" refers to the element $x^{*} \in X$ discussed in the definition. A target rule selects the alternative in between the agents votes that lies closest (in terms of $\leq^{*}$ ) to the target.

In Figure 4, we display the meet-semilattice corresponding to a typical target rule. Here, the alternative $x^{*}$ corresponds to $b$. The partial orders corresponding to target rules all have the "v"-shape as depicted here.

Theorem 4: A rule satisfies unanimity, anonymity, representative consistency, and strategyproofness if and only if it is a target rule. ${ }^{15}$

Proof: Clearly, the target rules are partial priority rules, and thus satisfy unanimity, anonymity, and representative consistency. It is well-known that they also satisfy strategy-proofness. Thus, we will show that any rule satisfying the axioms must be a target rule.

First, we show that for all $x, y, z \in X$, if $x \preceq y \preceq z$, then either $x \leq^{*} y \leq^{*} z$ or $z \leq^{*} y \leq^{*} x$. Call this property the chain property. Suppose that this statement is false. There are four alternative orderings of $x, y, z$ according to $\leq^{*}$. We will only

\footnotetext{
${ }^{13}$ As usual, for a binary relation $R, P$ denotes the asymmetric part of $R$. The alternative $x(R)$ is called the peak of $R$.

${ }^{14}$ In this environment, a rule selects only Pareto efficient alternatives if and only if it satisfies betweenness with respect to the peaks.

${ }^{15}$ For a fixed population $N \in \mathcal{N}$, it follows that the target rules must be generalized median voter rules, as defined in Moulin [15].
} 


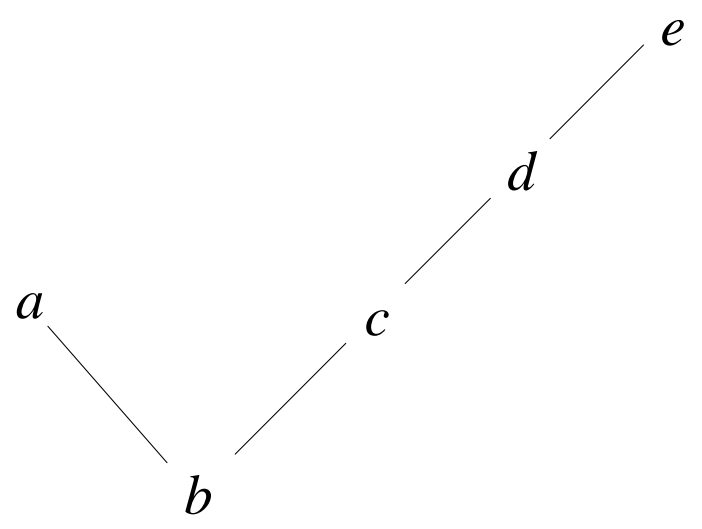

Figure 4: A target rule

show that one such ordering leads to an impossibility. The remaining three cases can be similarly proved. Thus, suppose $y \leq^{*} z \leq^{*} x$. Suppose, without loss of generality, that $y<^{*} z$ (this is without loss of generality, as otherwise, $z \leq^{*} y \leq^{*} x$ holds). Let $R \in S P\left(X, \leq^{*}\right)$ such that $x(R)=z$ and $x P y$. Then as $y \preceq z, f(x(R), y)=y$. However, $f(x, y)=x P y=f(x(R), y)$, in contradiction to strategy-proofness.

Next, we show that $f$ is an interval partial priority rule. Thus, let $x, y, z \in X$ such that $x, y \geq^{*} z$. If $x=y$, then clearly $x \wedge x=x$. So suppose that $x \neq y$, and suppose $x \wedge y<^{*} z$. Without loss of generality, suppose that $y>^{*} x$. Let $R \in S P\left(X, \leq^{*}\right)$ satisfy $x(R)=y$. Then $f(x, x)=x P(x \wedge y)=f(x(R), x)$, a contradiction to strategy-proofness. The case in which $x, y \leq^{*} z$ is proved similarly.

Let $x^{*} \equiv \bigwedge_{x \in X} x$. By definition of $x^{*}$, for all $x \in X, x^{*} \preceq x$. We prove that $i$ ) in the definition of target rule is true; $i i$ ) follows from a symmetric argument.

Let $y, z \in X$ such that $z \leq^{*} y \leq^{*} x^{*}$. We show that $z \succeq y$. As $f$ is an interval partial priority rule, $z \leq^{*} y \wedge z \leq^{*} y$. Moreover, by definition, $y \succeq y \wedge z \succeq x^{*}$, so that by the chain property and the fact that $x^{*} \geq^{*} y, x^{*} \geq^{*} y \wedge z \geq^{*} y$. Thus, $y \wedge z \leq^{*} y$ and $y \wedge z \geq^{*} y$, so that by the anti-symmetry of $\leq^{*}, y \wedge z=y$. Thus, by definition, $z \succeq y$.

To verify iii) in the definition of target rule, let $y, z \in X$ satisfy $y \leq^{*} x^{*} \leq^{*} z$. As $y \succeq y \wedge z \succeq x^{*}$, by the chain property, $x^{*} \geq^{*} y \wedge z \geq^{*} y$. As $z \succeq y \wedge z \succeq x^{*}$, by the chain property, $x^{*} \leq^{*} y \wedge z \leq^{*} z$. Therefore, $x^{*} \geq^{*} y \wedge z$ and $x^{*} \leq^{*} y \wedge z$. By the anti-symmetry of $\leq^{*}, y \wedge z=x^{*}$. 


\section{Discussion and conclusion}

\subsection{Previous literature related to representative consistency}

Representative consistency is mathematically not a new condition; however, we believe that the interpretation here is. Blackorby and Donaldson [5] study a condition called the "population substitution principle," which is the same as representative consistency. Their interest in the condition is in comparing the welfares of varying groups of agents. The literature on functional equations has studied this (dating back to 1930-see [13, 18], as well as [6]) and related notions. These works use the notion in a system of axioms to characterize the quasi-arithmetic means $[1,2]$. For a good introduction, see Diewert [7]. The main analytical distinction between our work and these works is that the cited works require the domain of a rule to be a continuum, usually the unit interval. To our knowledge, no other work has allowed the domain to be a finite set. The difference in the two domains leads to different results, as the continuum model admits a richer family of rules satisfying representative consistency. Of course, this is not to argue that representative consistency is unappealing in the finite model. The conceptual appeal of the condition as a primitive is independent of its analytical implications.

The condition of path independence, first formalized by Plott [19] also resembles representative consistency. However, path independence is a only well-defined for choice correspondences. Hence, representative consistency and path independence are not logically related. Theorem 5 of Plott [19] is closely related to our Corollary 1. Characterizations of the family of choice rules satisfying path independence are provided by Aizerman and Aleskerov [3], pp. 167-168.

Finally, the first works to study indirect vote aggregation in social choice models are those which study representative systems, mentioned in the introduction. This literature was introduced by Murakami [16, 17], and later studied by Fishburn [11, 12] and Fine [10]. These works introduce the distinction between direct and indirect voting in formal social choice models. Their interest is in understanding which voting rules can arise as representative systems when taking plurality rule as primitive and by allowing different voters and districts to have different "weights." The distinction is that they require no consistency between indirect and direct voting. Rather, they study which rules can be thought of as arising from indirect voting, where majority rule is applied at each level. Moulin [15], as also mentioned in the introduction, discusses the related idea of showing that a representative system of strategy-proof rules is itself strategy-proof.

\subsection{General comments}

Partial priority rules violate neutrality, which requires that the "names" of alternatives should not matter for a voting rule. In a pure voting model, anonymity and neutrality are incompatible when rules must be single-valued and environments with even populations of agents are permissible. A natural extension of our model would include rules which 
are allowed to be multi-valued. Such an extension requires redefining representative consistency for this case. Whether similar results obtain depends on this notion. Neutrality can also be captured when lotteries are permitted.

In our model, we implicitly assume that the same voting rule is applied at all levels of government. A natural extension of the model would study two different voting rules, say $f$ and $g$. The rule $f$ would be applied at the district level. The rule $g$ would be used for aggregating representative votes at the national level. In this scenario, representative consistency would say that the composition of $g$ with $f$ should always select the same alternative, independently of the partitioning of agents into districts. It turns out that if unanimity and anonymity are required for both voting rules, then the two voting rules coincide and Theorem 1 applies. To see why, let $N \in \mathcal{N}$ and let $x \in X^{N}$. By putting each agent into her own district, the outcome of the rule is $g\left(\left(f\left(x_{i}\right)\right)_{i \in N}\right)$. By putting all agents in the same district, the outcome of the rule is $g(f(x), \ldots, f(x))$. By unanimity of $f, g\left(f\left(x_{i}\right)_{i \in N}\right)=g(x)$, and by unanimity of $g, g(f(x), \ldots, f(x))=f(x)$. By representative consistency, $g\left(f\left(x_{i}\right)_{i \in N}\right)=g(f(x), \ldots, f(x))$, so that $g(x)=f(x)$. Hence $g=f$.

A natural question is whether Theorem 1 holds when the set of potential agents is finite. While we have no proof, we conjecture that if the cardinality of the set of potential agents is at least three, then a result akin to Theorem 1 holds. A potential approach to solving this problem is presented in Aczél [1] in his characterization of the quasi-arithmetic means based on the bisymmetry equation.

\section{Appendix A-Independence of the axioms}

The following are examples establishing the independence of our axioms. Each axiom is followed by a rule which violates it and satisfies the other two axioms of Theorem 1 .

Unanimity (constant rule): Let $f$ be a constant rule-i.e. there exists $z \in X$ such that for all $N \in \mathcal{N}$ and all $x \in X^{N}, f(x) \equiv z$.

Anonymity (dictatorship): Let $R$ be a linear order on the set of agents $\mathbb{N}$. For all $N \in \mathcal{N}$ and all $x \in X^{N}, f(x) \equiv x_{\arg \max _{R} N}$. Here, $\arg \max _{R} N$ refers to $\{i \in N: i R j$ for all $j \in N\}$. Thus, this rule selects that alternative voted for by the agent in $N$ with the highest priority.

Representative consistency (median voter): Let $\leq^{*}$ be a linear order on $X$. For all $N \in \mathcal{N}$ and all $x \in X^{N}$,

$$
f(x) \equiv \min _{\leq^{*}}\left\{x \in\left\{x_{i}\right\}_{i \in N}:\left|i: x_{i} \leq^{*} x\right| \geq \frac{|N|}{2}\right\} .
$$




\section{Appendix B-Equipopulous districts}

This appendix discusses a restricted version of representative consistency.

Weak representative consistency: For all $N \in \mathcal{N}$, all partitions $\left\{N_{1}, \ldots, N_{m}\right\}$ of $N$ such that for all $i, j,\left|N_{i}\right|=\left|N_{j}\right|$, and all $x \in X^{N}, f(x)=f\left(f\left(x_{N_{1}}\right)^{N_{1}}, \ldots, f\left(x_{N_{m}}\right)^{N_{m}}\right)$.

As the name suggests, weak representative consistency is a weaker condition than representative consistency. Under unanimity, weak representative consistency has no bite in environments in which the set of agents cannot be partitioned into nontrivial districts of equal cardinalities. This is the case if the set of agents has a cardinality which is a prime number.

We establish that no new rules emerge when requiring that districts be equipopulous. Thus, even if all districts are required to contain the same number of agents, any rule which is not a partial priority rule presents some opportunities for gerrymandering.

Theorem 5 generalizes Theorem 1 using the weak representative consistency condition.

Theorem 5: A rule satisfies unanimity, anonymity, and weak representative consistency if and only if it is a partial priority rule.

The theorem relies on the following lemma, whose proof is identical to Lemma 1. Thus, we state it without proof.

Lemma 2: If a rule satisfies unanimity, anonymity, and weak representative consistency, then it satisfies replication invariance.

The next lemma establishes that if a rule satisfies unanimity, weak representative consistency, and replication invariance, then it satisfies representative consistency. Taken together, Lemmas 2 and 3 will directly imply Theorem 5.

Lemma 3: If a rule satisfies unanimity, weak representative consistency, and replication invariance, then it satisfies representative consistency.

Proof: Let $f$ be a rule that satisfies unanimity, weak representative consistency, and replication invariance. Let $N \in \mathcal{N}$ and let $x \in X^{N}$. Let $\left\{N_{1}, \ldots, N_{m}\right\}$ be a partition of $N$. We claim that $f(x)=f\left(f\left(x_{N_{1}}\right)^{N_{1}}, \ldots, f\left(x_{N_{m}}\right)^{N_{m}}\right)$.

By replication invariance,

$$
f(x)=f\left(\prod_{i=1}^{m}\left|N_{i}\right|\right)
$$


Rewrite

$$
\left.f\left(\prod_{i=1}^{m}\left|N_{i}\right|\right)=f\left(\left(x_{N_{k}}\right)^{\prod_{i=1}^{m}\left|N_{i}\right|}\right)_{k=1}^{m}\right) .
$$

Again, rewrite

$$
\left.f\left(\left(x_{N_{k}}\right)^{m} \prod_{k=1}^{m}\right)_{\left|N_{i}\right|}^{m}\right)=f\left(\left(\left(\prod_{i \neq k}\left|N_{i}\right|\right)^{\left|N_{k}\right|}\right)_{k=1}^{m}\right) .
$$

For all $k=1, \ldots, m, \prod_{N_{k}}^{\prod_{i \neq k}\left|N_{i}\right|}$ has cardinality $\prod_{i=1}^{m}\left|N_{i}\right|$. By weak representative consistency,

$$
\left.\left.f\left(\left(\prod_{i \neq k}\left|N_{i}\right|\right)^{\left|N_{k}\right|}\right)_{k=1}^{m}\right)=f\left(f\left(\prod_{i \neq k}\left|N_{i}\right|\right)^{\left|N_{k}\right|} \prod_{i=1}^{m}\left|N_{i}\right|\right)_{k=1}^{m}\right) .
$$

By replication invariance, for all $k=1, \ldots, m$,

$$
f\left(\prod_{\substack{i \neq k \\ N_{k}}}\left|N_{i}\right|\right)=f\left(x_{N_{k}}\right)
$$

Thus,

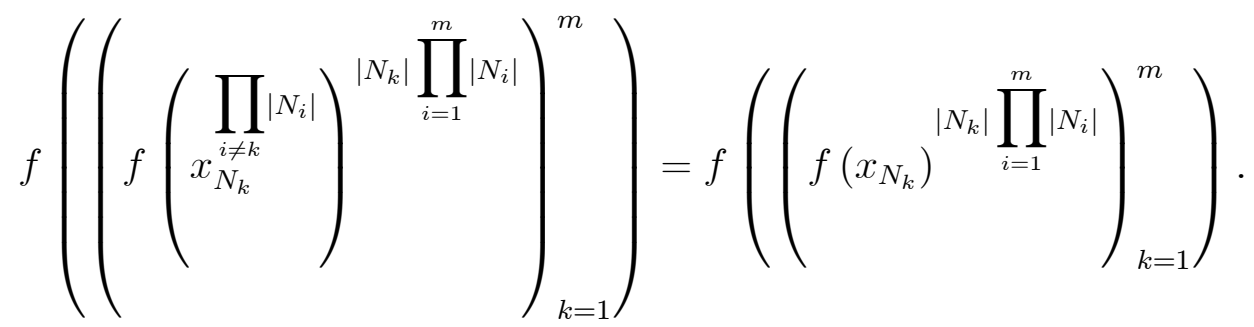

By replication invariance,

$$
f\left(\left(f\left(x_{N_{k}}\right)^{\left|N_{k}\right|} \prod_{i=1}^{m}\left|N_{i}\right|\right)_{k=1}^{m}\right)=f\left(\left(f\left(x_{N_{k}}\right)^{\left|N_{k}\right|}\right)_{k=1}^{m}\right) .
$$


By anonymity, which is implied by replication invariance,

$$
f\left(\left(f\left(x_{N_{k}}\right)^{\left|N_{k}\right|}\right)_{k=1}^{m}\right)=f\left(f\left(x_{N_{1}}\right)^{N_{1}}, \ldots, f\left(x_{N_{m}}\right)^{N_{m}}\right) .
$$

Thus, $f(x)=f\left(f\left(x_{N_{1}}\right)^{N_{1}}, \ldots, f\left(x_{N_{m}}\right)^{N_{m}}\right)$ and representative consistency is satisfied.

The proof of Theorem 5 is now simple.

Proof: Let $f$ be a partial priority rule. Then it clearly satisfies unanimity, anonymity, and weak representative consistency.

Let $f$ be a rule that satisfies unanimity, anonymity, and weak representative consistency. By Lemma $2, f$ satisfies replication invariance. By Lemma $3, f$ satisfies representative consistency. Thus, $f$ satisfies unanimity, anonymity, and representative consistency. By Theorem 1, $f$ is a partial priority rule.

\section{References}

[1] Aczél, J. (1966): Lectures on Functional Equations and their Applications, Academic Press, New York

[2] Aczél, J. (1997): "Bisymmetry and consistent aggregation: Historical review and recent results," in Choice, Decision, and Measurement: Essays in Honor of $R$. Duncan Luce, (A.A.J. Marley, Ed.), Lawrence Erlbaum Associates, New Jersey, 225-233.

[3] Aizerman, M., and F. Aleskerov (1995): Theory of Choice, Elsevier Science, Amsterdam.

[4] Birkhoff, G. (1967): Lattice Theory, third edition, American Mathematical Society, Providence.

[5] Blackorby, C., and D. Donaldson (1984): "Social criteria for evaluating population change," Journal of Public Economics 25, 13-33.

[6] de Finetti, B. (1931): "Sul concetto di media," Giornale dell' Instituto Italiano degli Attuari 2, 369-396.

[7] Diewert, W.E., (1993): "Symmetric means and choice under uncertainty," in Essays in Index Number Theory, Vol. 1, (W.E. Diewert and A.O. Nakamura, Eds.), pp. 355433, Elsevier Science Publishers, B.V.

[8] Duggan, J., and C. Martinelli (2001): "A Bayesian model of voting in juries," Games and Economic Behavior 37, 259-294.

[9] Feddersen, T. and W. Pesendorfer, (1998): "Convicting the innocent: the inferiority of unanimous jury verdicts," American Political Science Review 92, 23-35. 
[10] K. Fine, (1972): "Some necessary and sufficient conditions for representative decision on two alternatives," Econometrica 40, 1083-1090.

[11] Fishburn, P.C., (1971): "The theory of representative majority decision," Econometrica 39, 273-284.

[12] Fishburn, P.C., (1973): The Theory of Social Choice, Princeton University Press, New Jersey.

[13] Kolmogorov, A., (1930): "Sur la notion de la moyenne," Rendiconti Accademia dei Lincei (6) 12, 388-391.

[14] May, K.O., (1952): "A set of independent necessary and sufficient conditions for simple majority decision," Econometrica 20, 680-684.

[15] Moulin, H., (1980): "On strategy-proofness and single peakedness," Public Choice $35,437-455$.

[16] Murakami, Y., (1966): "Formal structure of majority decision," Econometrica 34, 709-718.

[17] Murakami, Y., (1968): Logic and Social Choice, Dover, New York.

[18] Nagumo, M., (1930): "Uber eine Klasse der Mittelwerte," Japan Journal of Mathematics 7, 71-79.

[19] Plott, C. (1973): "Path independence, rationality, and social choice," Econometrica 41, 1075-1091.

[20] Thomson, W., (1993): "The replacement principle in public good economies with single-peaked preferences," Economics Letters 42, 31-36.

[21] Thomson, W., and S. Ching, (1993): "Population-monotonic solutions in public good economies with single-peaked preferences," mimeo, University of Rochester.

[22] Young, H.P., (1975): "Social choice scoring functions," SIAM Journal of Applied Mathematics 28, 824-838. 